[18] Watkins, R. R. (2012). Investigating the association between vitamin D deficiency and sepsis: challenges and future prospects. Expert Review of Anti-Infective Therapy, 10 (7), 723-725. doi: 10.1586/eri.12.61

[19] Baczyńska-Strzecha, M., Kalinka, J. (2017). Assessment of correlation between vitamin D level and prevalence of preterm births in the population of pregnant women in Poland. International Journal of Occupational Medicine and Environmental Health. doi: 10.13075/ijomeh.1896.01146

[20] Flynn, L., Zimmerman, L. H., McNorton, K., Dolman, M., Tyburski, J., Baylor, A. et. al. (2012). Effects of vitamin D deficiency in critically ill surgical patients. The American Journal of Surgery, 203 (3), 379-382. doi: 10.1016/j.amjsurg.2011.09.012

\title{
EVALUATION OF THE THERAPEUTIC AND PREVENTIVE POTENTIAL OF THE MEDICINAL PRODUCT CANEPHRON N IN THE TREATMENT OF GESTATIONAL PYELONEPHRITIS IN PREGNANT WOMEN
}

\author{
Roman Sheremeta \\ Department of Urology \\ Regional Clinical Hospital \\ 4 Nekrasova str., Lviv, Ukraine, 79010 \\ sheremetar@ukr.net \\ Maksim Sabadash \\ Department of Urology \\ Lviv Regional Oncology Treatment and Diagnostic Center \\ 2 a Hasheka str., Lviv, Ukraine, 79031 \\ maxsabadash@i.ua \\ Alexander Shulyak \\ Institute of Urology of National Academy of Medical Sciences of Ukraine \\ $9 A$ Vynnychenko str., Kyiv, Ukraine, 04053 \\ avshulyak@hotmail.com
}

\footnotetext{
Abstract

Background: Gestational pyelonephritis (GP) is one of the most common and serious diseases of the middle and second half of pregnancy, which complicates approximately $6 \%$ of pregnancies, and is one of the leading causes of non-obstetric pre-delivery hospitalization.

Methods. The paper provides data from the open, multicentre, prospective, comparative (parallel group) study in 60 pregnant women diagnosed with gestational pyelonephritis, which were divided into two groups 30 subjects each. Patients in the treatment group received antibacterial therapy for 7 days + treatment with standard dosage of medicinal product Canephron $\mathrm{N}$ for 3 months. Patients in the control group received antibiotic therapy alone for 7 days.

Results. The average age of pregnant women at the time of diagnosing was $23 \pm 6.23$ years; it was the first pregnancy for 39 women (65\%). The most common complaints at admission to the in-patient department were as follows: lumbar pain (96.2 \%), dysuria (70.2\%), rise in temperature above $38^{\circ} \mathrm{C}(64.4 \%)$. Leukocyturia and bacteriuria were detected in $100 \%$ of cases with prevalence of E. coli in $84.2 \%$ (101 of 120) of cases. By 30 day in GP patients in the treatment group bacteriuria was detected in $2 / 30(6.67 \%)$ patients, and in the control group - in 5/30 (16.7\%) patients (p 1: $2<0.05)$, by 60 day in the treatment group $2 / 30(6.67 \%)$, in the control group - 8/30 (26.7\%) (p 1: $2<0.05)$, respectively, by 90 day $-3 / 30(10 \%)$ and $10(33.3 \%)$, respectively (p 1: $2<0.05)$. The disease relapsed in 1 of $30(3.33 \%)$ GP patients in the treatment group and 3/30 (10\%) patients in the control group (p 1: $2<0.05)$ and 3/30 (10\%) patients in the treatment group with CP, and 7/30 (23.3\%) patients in the control group during the follow-up period (p 1: $2<0.05$ ).
} 
The use of the herbal medicinal product Canephron $\mathrm{N}$ in pregnant women with gestational pyelonephritis and in the acute exacerbations of chronic pyelonephritis has shown its high efficacy and undoubted therapeutic and prophylactic effect, good tolerability and decrease in the frequency of relapses of pyelonephritis.

Keywords: gestational pyelonephritis, pregnancy, Canephron N, leukocyturia, bacteriuria, urosepsis, kidney, infection, urinary tract.

\section{Introduction}

Gestational pyelonephritis (GP) is an acute inflammatory infection affecting mainly the interstitial tissue, tubular apparatus, and the walls of the renal collecting system, with the onset during the current pregnancy [1,2]. GP is one of the most common and serious diseases of the middle and second half of pregnancy, which complicates approximately $6 \%$ of pregnancies, and is one of the leading causes of non-obstetric pre-delivery hospitalization $[3,4]$. In the past, epidemiological data showed that the incidence of gestational pyelonephritis (GP) in pregnant women reached $10 \%$ [5], but with the improvement of pre-delivery observation, the incidence of acute pyelonephritis has decreased in recent years. Currently, the term "acute and chronic gestational pyelonephritis" is used, which includes pyelonephritis developing during pregnancy, childbirth, and in the postpartum period. The symptoms of acute pyelonephritis in pregnant women are the same as in secondary acute pyelonephritis of other aetiology. The onset of the disease is acute; it is often characterized with a severe course with intoxication. The clinical manifestations include pain in the affected kidney, hectic rise of body temperature, the following are often observed: headache, general weakness, significant deterioration of general condition, chills. Sometimes there are symptoms of renal colic. Urosepsis may develop [6, 7].

GP occurs more often in the first than in repeated birth, and has a negative effect on the course of pregnancy and foetal condition (high abortion incidence, anaemia, early gestoses and preeclampsia, growth retardation, and foetal distress) [3], and the new-borns often have the signs of intrauterine infection. In addition, such children are more susceptible to purulent and septic diseases in the postnatal period of life [4]. The frequency of asymptomatic bacteriuria does not increase during pregnancy compared with non-pregnant individuals [8], but physiological and anatomical changes that cause urinary stasis during pregnancy increase the risk of development of the clinical manifestations of the disease [9], especially in the middle of pregnancy [10], and more commonly on the right $[3,7]$. Changes in the endocrine profile in pregnant women have a certain negative effect on the urinary tract. Obviously, the pregnancy hormones, which are active from the very beginning of pregnancy, affect the tonus of the entire urinary tract in the first months of pregnancy, but enlargement of the uterus mechanical factors play a certain role, disturbing the urine outflow from the kidneys $[2,11]$.

It should be noted that gestational pyelonephritis has a negative effect not only on the body of the mother, but also on the foetus: it promotes the development of foetal hypotrophy, reduction of body weight of the new-born, increases the risk of stillbirth.

Treatment of PN in pregnant women is currently quite aggressive, in particular, all patients are hospitalized to an in-patient department and undergo antibiotic therapy until afebrile rates within 24 hours and improved clinical symptoms are achieved $[12,20]$ to minimize kidney damage and adverse effects on the foetus.

After gestational pyelonephritis, the kidney function remains low in $20 \%$ of women [7]. In gestational period it is important to use medicinal products that do not cause embryotoxic effect, such herbal preparations $[5,11]$. Physicians may take advantage of the medicinal product Canephron $\mathrm{N}$, which is especially popular due to its herbal nature, good tolerance, absence of adverse effects on the foetus, and a fairly extensive and successful use in obstetrics [3].

\section{Aim of research}

Improve the treatment of patients with gestational pyelonephritis with the help of the herbal medicinal product Canephron N. 


\section{Materials and methods}

Open label, single centre, prospective, comparative (parallel group) study.

The study observed 60 pregnant women with inflammatory diseases of the upper urinary tract: acute gestational pyelonephritis $(n=60)$. Depending on the chosen treatment, the patients were divided into 2 groups: the treatment group $(n=30)$ received antibiotic therapy to eliminate clinical symptoms and laboratory manifestations for 7 days + Canephron $\mathrm{N}$ in standard dosages with subsequent switch to Canephron $\mathrm{N}$ within 3 months to prevent the relapse of the inflammatory infection.

Control group $(n=30)$ : received standard antibiotic therapy to eliminate clinical symptoms and laboratory manifestations for 7 days without further therapy with Canephron N.

Canephron $\mathrm{N}$ was prescribed as a solution (aqueous-alcohol extract) at the dose of 50 drops 3 times daily or 2 tablets 3 times daily for 30 days.

Patients were chosen by randomization. In order to evaluate the effectiveness of rehabilitation and prevention measures, the study included pregnant women with PN, which developed before gestational 30 week.

Inclusion criteria: documented diagnosis of gestational pyelonephritis. Age of subjects: $18-45$ years.

Exclusion criteria: malformations of the urinary tract, individual intolerance to the components of the medicinal product Canephron N, presence of coral calculi and concretions affecting the urodynamics, diabetes mellitus, chronic renal failure, and absence of consent to participate in the study.

These groups were comparable in age (19 to 35 years), parity, presence of somatic pathology, and duration of hospitalization. All pregnant women were consulted by a physician, urologist, gynaecologist, and nephrologist. Treatment took place at the in-patient department.

Clinical examination methods were as follows: complete blood count and urinalysis, serum creatinine count and serum urea, and ultrasound. Culture was taken at admission, on day 14, day 35 , and day 90 of therapy. Urine for culture and sensitivity to antibacterial drugs was obtained with the help of Nelaton 6 Fr.catheter, clinically significant bacteriuria value was $>10^{5} \mathrm{CFU}$ of uropatogen $/ \mathrm{mL}$ in urine.

The ultrasonography of the kidneys was performed with recording of the pulsation index (PI) and the resistance index (RI) in the renal artery and in the segmental, interlobular and arcuate arteries of the kidney. Ultrasonography and, if necessary, an NMR imaging recommended by the EAU (2015, grade B) for pregnancy testing due to the minimal impact of these methods on foetal development. X-ray and radioisotope tests are contraindicated in pregnant women because of the possible harmful effects on the foetus. Endoscopic studies are undesirable, as well.

Statistical processing of variation series included the calculation of mean values (M) and mean errors (m). Comparison of variation series was carried out using the Student's t-test.

\section{Result}

The average age of pregnant women at the time of diagnosing was $23 \pm 6.23$ years ( \pm mean standard deviation), it was the first pregnancy for 39 women $(65 \%)$.

Pregnant women with acute inflammation in the kidneys (60) were hospitalized to the in-patient department during the following periods of gestation: week 12-16 - 11 (17.5\%) cases, week 16-22 - $22(36.7 \%)$ cases, week 23-30 - 27 (45.8\%) cases. At the time of admission all patients received cephalosporin antibiotics, and once culture was obtained, the pregnant women proceeded with the latter or switched to another antibacterial preparation within 7 days, based on their sensitivity.

The most common complaints at admission to the in-patient department were as follows: lumbar pain (96.2\%), dysuria (70.2\%), rise in temperature above $38 \mathrm{C}$ (64.4\%), chills (31.9\%), and other symptoms (50.3\%). According to urinalysis, pyuria was present in $100 \%$ of cases, and microscopic haematuria - in $38.2 \%$ of cases.The average haemoglobin values at admission were $111 \pm 6.7 \mathrm{~g} / \mathrm{L}$. The average leukocyte count in the blood was $12.1 \pm 4.6 \times 109 / \mathrm{L}$. The average body temperature at admission was $37.5 \pm 0.9$ Celsius and the Pasternatsky's symptom (Costovertebral angle 
tenderness (CVAT)) was more often positive on the right side in 76 of 120 cases $(63.3 \%)$ than on the left side (29/120, 24.2\%), and was bilateral in 15 cases (12.5\%). Clinical and laboratory parameters of the patient distribution in the two groups are given in Table 1.

Table 1

Clinical and laboratory parameters in two groups of patients

\begin{tabular}{ccc}
\hline Parameters & Treatment group $(\mathbf{n}-\mathbf{3 0})$ & Control group (n-30) \\
\hline Average age & $22 \pm 5.22$ & $24 \pm 4.18$ \\
Lumbar pain & $92.3 \%$ & $94.6 \%$ \\
Abdominal pain & $85.7 \%$ & $83.5 \%$ \\
Dysuria & $71.2 \%$ & $69.8 \%$ \\
Fever $>38.0^{\circ} \mathrm{C}$ & $65.1 \%$ & $63.9 \%$ \\
Chills & $51.4 \%$ & $52.2 \%$ \\
Pyuria & $100 \%$ & $100 \%$ \\
Microhematuria & $37.8 \%$ & $38.6 \%$ \\
Haemoglobin & $109 \pm 4.7$ & $112 \pm 6.3$ \\
Leukocytosis & $12.3 \pm r, \mathrm{~V}$ & $11.8 \pm 5.3$ \\
ESR & $36 \pm 8.2$ & $39 \pm 6.4$
\end{tabular}

At admission all patients underwent obstetric ultrasound scan, and all of them were normal. In $78(65 \%)$ patients, according to the ultrasound scan data, normal ureteral patency was observed, in $43(35.8 \%)$ cases ureterohydronephrosis was observed, 12 of which had renal stents installed by the end of delivery, in the rest of cases of ureterohydronephrosis the knee-elbow position was sufficient.

Bacteriuria was detected in $100 \%$ of patients with prevalence of E. coli in $84.2 \%$ (101 of $120)$ of cases; other organisms were Klebsiella Spp. (9.17\%, 11 of 120) and Proteus Spp. (6.67 \% 8 of 120), which were most sensitive to ceftriaxone ( $81.55 \%$ of cases), cefazolin (79.5\%), amoxicillin + clavulanic acid (77.1\% of cases), cefotaxime (72.6\%), gentamicin $(60.57 \%$ of cases).

Following the distribution into groups, the women in the treatment group proceeded with the treatment with Canephron ${ }^{\circledR} \mathrm{N}$ for the purposes of rehabilitation, no treatment was prescribed in the control group. By 10 day in the treatment group with GP bacteriuria was detected in 3 of $30(10 \%)$ patients, and in the control group - in 4 of $30(13.3 \%)$ GP patients (p 1: $2>0.05)$, by 30 day in the treatment group of GP patients bacteriuria was detected in $2 / 30(6.67 \%)$ patients, and in the control group - in 5/30 (16.7\%) patients ( $1: 2<0.05)$, by 60 day in the treatment group $2 / 30(6.67 \%)$, in the control group $-8 / 30(26.7 \%)(\mathrm{p} 1: 2<0.05)$, respectively, by 90 day $3 / 30(10 \%)$ and $10(33.3 \%)$, respectively (p 1: $2<0.05)$. Data on bacteriuria in patients in two GP groups are shown in Fig. 1.

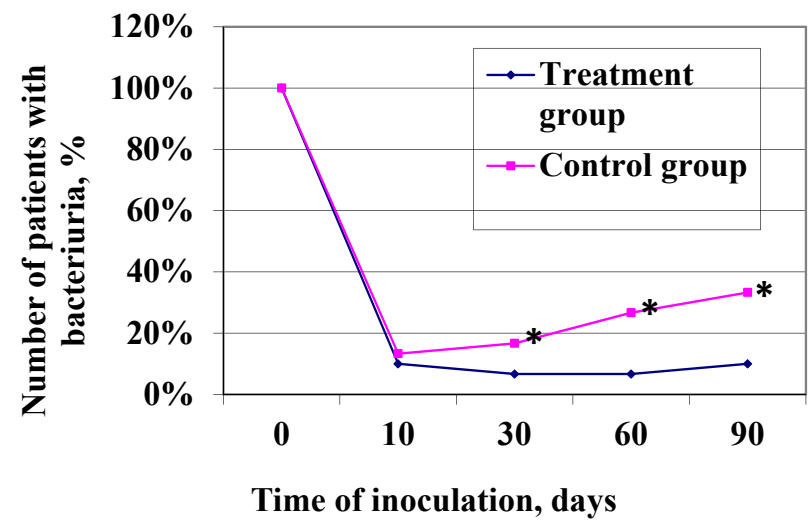

Fig. 1. Data on bacteriuria in patients in two GP groups: ${ }^{*}-\mathrm{p}<0.05$ 
According to the above table, in GP patients in the treatment group by 30 day the number of patients with bacteriuria was by 2-fold lower compared to the control group, by 4-fold lower by 60 day, and by 3 -fold lower by 90 day, respectively.

The disease relapsed in 1 of $30(3.33 \%)$ GP patients in the treatment group and 3/30 (10\%) patients in the control group (p 1: $2<0.05)$ and $3 / 30(10 \%)$ patients in the treatment group with $\mathrm{CP}$, and 7/30 (23.3\%) patients in the control group during the follow-up period (p 1: $2<0.05)$. PN relapses in patients in two groups with GP and CP are shown in Fig. 2.

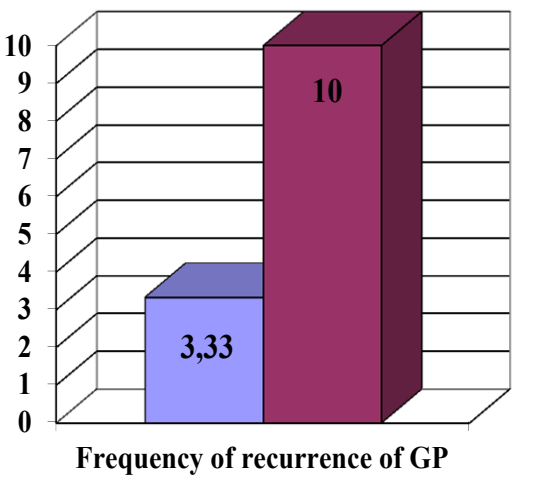

\section{$\square$ The main group \\ $\square$ The control group}

Fig. 2. PN relapses in patients in two groups with GP during the follow-up period: $*-p<0.05$

The above table shows that PN relapses were significantly lower in patients of the treatment group $(\mathrm{p}<0.05)$.

Based on the data of the dopplerographic examination of the renal arteries, the pathological increase in the values of the pulsation index (PI) $(1.63 \pm 0.15)$ and the index of resistance (IR) $(0.73 \pm 0.12)$ was observed in acute pyelonephritis, the increase in the velocity of blood flow was observed at the level of the renal artery and reached $417 \pm 29 \mathrm{~mL}$. $/ \mathrm{min}$. Antibacterial therapy contributed to the recovery of renal blood flow and normalization of the parameters of IR and PI in both study groups ( $1: 2>0.5)$. After 30 days from the beginning of treatment of PN, in the treatment group the IR $(0.59 \pm 0.04)$ and PI $(1.12 \pm 0.13)$ values were within the physiological limits, while in the control group the IR $(0.71 \pm 0.09)$ and PI $(1.33 \pm 0.12)$ significantly exceeded the values of the treatment group, and their level indicated a high risk of relapse (p1: $2<0.05$ ). The velocity of blood flow at the level of the renal artery in the treatment group (Canephron N) was $283 \pm 28 \mathrm{~mL} / \mathrm{min}$, and in the control group $-302 \pm 32 \mathrm{~mL} / \mathrm{min}(\mathrm{p} 1: 2>0.5)$. After PN the risk of miscarriage was observed in $9(7.5 \%)$ pregnancies, the risk of preterm labour was diagnosed in $14(11.7 \%)$ pregnancies, which occurred in 3 cases $(2.5 \%)$. There were no side effects in pregnant women after administration of Canephron $\mathrm{N}$, the patients mentioned good tolerability of the medicinal product.

\section{Discussion}

According to Patterson TF 1997 et al., the frequency of asymptomatic bacteriuria does not increase during pregnancy compared with non-pregnant women [4], but physiological and anatomical changes that cause urinary stasis during pregnancy increase the risk of clinical manifestations of the disease [17], especially in the middle of pregnancy [16], and more commonly on the right [7].

The average age of pregnant women at the time of diagnosing was $23 \pm 6.23$ years, for 39 women (65\%) this was the first pregnancy, which was slightly less than in the Gilstrap LC 1991 study, in which $75 \%$ of the cases were first-borns [9].

Peculiarities of the disease pathogenesis suggest that neurohumoral factors along with the mechanical pressure of the enlarged uterus on the ureter play the key role in the occurrence of urinary hypotonia and urodynamic disorders. Hypotonia of the ureter combined with their obstruction by the enlarged uterus leads to urinary stasis, urinary stagnation in the ureter and the renal collecting system, and contributes to infection $[14,15]$. 
In their studies Gilstrap LC et al. 2001 and Millar LK et al. 2003 showed maternal and foetal morbidity $[8,11]$ associated with pyelonephritis, fever in the first trimester of pregnancy during organogenesis was associated with teratogenicity, miscarriage and premature delivery.

In our study, E.Coli was cultured in $84.2 \%$ (101 of 120) of cases, which corresponds to the previous international studies by Faundes A. et al. 2008 and Fan Y. D. et al. 2011 [7, 18] with $82 \%$ sensitivity to ceftriaxone. GP treatment begins with the recovery of urine outflow from the kidney. For this positional therapy is used: patients are recommended to lie on the "healthy" side, with a raised bed on the side of the legs, to take the knee-elbow position. Sometimes this simple procedure alone leads to a reduction in the acuity of the kidney inflammation due to a decrease in the pressure of the enlarged uterus on the ureter. When the indicated conservative therapy is ineffective, and when the examination reveals a disorder in the urine outflow from the affected kidney, stenting of the ureter may be used. Typically, a stenting catheter is delivered to the renal pelvis without interference through the entire ureter, and the discharge of urine from the stenting catheter in frequent droplets or even in a stream starts at the height of $13-20 \mathrm{~cm}$ from the bladder, that is precisely at the level where the uterus presses the ureter to the narrowest area of bone pelvic ring. It is advisable to leave the stenting catheter for several weeks after delivery.

In parallel, if necessary, antibiotic therapy is used, for which the least toxic antibacterial drugs are chosen, especially before pregnancy 12 week. After pregnancy week 12, antibiotics may be used more widely, including aminoglycosides, etc. This is due to the fact that the anlage of the main body organs and systems is completed by the end of 12 week.

For the treatment of acute pyelonephritis in pregnant women, the EAU (2015, grade A) recommends the following: ceftriaxone $1-2 \mathrm{~g}$ IV or IM every 24 hours, aztreonam $1 \mathrm{~g}$ IV every 812 hours, piperacillin-tazobactam 3.375-4.5 g IV every 6 hours, cefepime $1 \mathrm{~g}$ IV every 12 hours, imipenem/cilastatin $500 \mathrm{mg}$ IV every 6 hours, ampicillin 2 g IV every 6 hours, gentamicin $3-5 \mathrm{mg} / \mathrm{kg} /$ day IV at 3 doses.

Thus, today even acute severe pyelonephritis in pregnant women can be eliminated and is not considered to be an obstacle to a favourable pregnancy outcome and delivery. In most women, physiological changes on the part of the urinary tract due to pregnancy resolve after delivery without any consequences. Yet, in those who had acute pyelonephritis, urodynamic disorders do not resolve entirely in all cases. The most common consequence of this disease is chronic pyelonephritis, rarely - kidney stone disease (urolithiasis), and other diseases.

Consequently, pregnant women who have suffered from pyelonephritis, are significantly more likely to develop kidney disease due to a prolonged urodynamics disorder, in which postnatal pyelonephritis is detected in $25-30 \%[16,19]$. Gestational pyelonephritis may develop in the postpartum period in the first days or weeks after childbirth, when the tonus of the urinary tract has not fully recovered.

The risk factors for the development of postpartum pyelonephritis include: pregnancy hormones (3 months) and dilated urinary tract (approximately 1.5 months); inflammatory diseases of the genital organs; complications in the postpartum period (hypotonia of the uterus, bleeding, etc.). In cases of post-natal pyelonephritis, a detailed urological examination is carried out, including $\mathrm{X}$-ray and radioisotope studies, the purpose of which is to determine the state of kidneys and the urinary tract, as well as to identify or rule out any other causes of pyelonephritis - abnormalities of the urinary system, tuberculosis, kidney stones.

Pazos Otero N et al. (2007) have presented quite interesting results having studied the data from 4,700 pregnant women in 2004. The incidence rate of pyelonephritis was $0.21 \%$, with E.coli being the only pathogen inoculated in all cases. In all cases, the prognosis was favourable [17].

Measures aimed at prevention of acute pyelonephritis in pregnant women are focused on two directions: preventive treatment of chronic urinary infection and the improvement of the outflow of urine from the kidneys by means of positional exercises: the periodic taking the knee-elbow position by the patient; the patient is also advised to lie only on the side opposite to the principal expansion of the renal pelvis, with a raised bed on the side of the legs. 
When performing such positional exercises, it is important that the patient is professionally instructed by the physician and the midwife observing the pregnant woman. In all the above mentioned positions, the pressure of the uterus on the urinary tract decreases, the urine outflow improves, the dilation of the renal pelvis decreases significantly. This is confirmed by ultrasonography, which is preferably used to control the prevention of pyelonephritis in pregnant women.

Gilstrap LC at all 2001 has reported that disease recurrence is observed in one-third of pregnant women with chronic urinary tract infections [9]. In our study, disease recurrence was observed in $10 \%$ of patients with chronic pyelonephritis during the 90 -day follow-up period.

Thus, the combination of antimicrobial and anti-inflammatory effects of the medicinal product Canephron $\mathrm{N}$ made it possible to significantly reduce the bacteriuria and the rate of disease recurrence in patients with gestational pyelonephritis and exacerbation of chronic pyelonephritis. Canephron $\mathrm{N}$ is an efficient herbal agent for treatment of inflammatory diseases of the urinary tract in pregnant women as it has a standardized composition, an optimal complex of effects required to treat chronic urinary tract infections in pregnant women. Most significantly, the diverse and wide range of herbal substances with antibacterial activity is significantly preventive in the development of bacterial resistance, and determines the efficacy of Canephron $\mathrm{N}$ used in the long-term preventive therapy.Yet, the absolute safety of administration of this herbal medicinal product in patients with urinary tract infections, and especially in pregnant women, is the primary advantage of Canephron N.

\section{Conclusions}

1. The medicinal product Canephron $\mathrm{N}$ reliably reduces bacteriuria in patients with GP by 3 -fold in the treatment group by 30 day $(2 / 30 ; 6.67 \%)$ vs. the control group $(5 / 30,16.7 \%)$, by 4 -fold by 60 day in the treatment group $(2 / 30 ; 6,67 \%)$ vs. the control group $(8 / 30 ; 26,7 \%) 5)$, and by 3 -fold by 90 day $-3 / 30(10 \%)$ and $10(33.3 \%)$ respectively.

2. The plant-based medicinal product Canephron $\mathrm{N}$ significantly reduces the rate of relapse of the disease $(1 / 30,3.33 \%)$ in GP patients in the treatment group $(3 / 30,10 \%)$ vs. the patients in the control group by 3 -fold during the follow-up period.

\section{References}

[1] Ailamazyan, E. K., Kulakov, V. I., Radzinsky, V. E., Savelyeva, G. M. (2009). Obstetrics. National Guidelines. Moscow: "GEOTAR-Media”, 1200.

[2] Gilstrap, L. C., Cunningham, F. G., Whalley, P. J. (1981). Acute pyelonephritis in pregnancy: an anterospective study. Obstetrics and Gynecology, 57 (4), 409-413.

[3] Hammond, N. A., Nikolaidis, P., Miller, F. H. (2012). Infectious and Inflammatory Diseases of the Kidney. Radiologic Clinics of North America, 50 (2), 259-270. doi: 10.1016/j.rcl.2012.02.002

[4] Stevens, P. E. (2013). Evaluation and Management of Chronic Kidney Disease: Synopsis of the Kidney Disease: Improving Global Outcomes 2012 Clinical Practice Guideline. Annals of Internal Medicine, 158 (11), 825. doi: 10.7326/0003-4819-158-11-201306040-00007

[5] Kulakova, V. I., Serov, V. N. (2008). Medicinal products used in obstetrics and gynaecology. Moscow: "GEOTAR-Media", 384.

[6] Kenzaka, T., Kato, K., Kitao, A., Kosami, K., Minami, K., Yahata, S. et. al. (2015). Hyperammonemia in Urinary Tract Infections. PLOS ONE, 10 (8), e0136220. doi: 10.1371/journal.pone.0136220

[7] Tessema, J., Jefferds, M. E., Cogswell, M., Carlton, E. (2009). Motivators and Barriers to Prenatal Supplement Use among Minority Women in the United States. Journal of the American Dietetic Association, 109 (1), 102-108. doi: 10.1016/j.jada.2008.10.013

[8] Kumar, S., Dave, A., Wolf, B., Lerma, E. V. (2015). Urinary tract infections. Disease-a-Month, 61 (2), 45-59. doi: 10.1016/j.disamonth.2014.12.002

[9] Wing, D. A., Rumney, P. J., Preslicka, C. W., Chung, J. H. (2008). Daily Cranberry Juice for the Prevention of Asymptomatic Bacteriuria in Pregnancy: A Randomized, Controlled Pilot Study. The Journal of Urology, 180 (4), 1367-1372. doi: 10.1016/j.juro.2008.06.016

[10] Hill, J. B., Sheffield, J. S., McIntire, D. D., Wendel, G. D. (2005). Acute Pyelonephritis in Pregnancy. Obstetrics \& Gynecology, 105 (1), 18-23. doi: 10.1097/01.aog.0000149154.96285.a0 
[11] Dalal, S., Nicolle, L., Marrs, C. F., Zhang, L., Harding, G., Foxman, B. (2009). Long-TermEscherichia coliAsymptomatic Bacteriuria among Women with Diabetes Mellitus. Clinical Infectious Diseases, 49 (4), 491-497. doi: 10.1086/600883

[12] Oktar, S. Ö., Yücel, C., Özdemir, H., Karaosmanoglu, D. (2004). Doppler Sonography of Renal Obstruction. Journal of Ultrasound in Medicine, 23 (7), 929-936. doi: 10.7863/jum.2004.23.7.929

[13] Geerlings, S. E. (2008). Urinary tract infections in patients with diabetes mellitus: epidemiology, pathogenesis and treatment. International Journal of Antimicrobial Agents, 31, 54-57. doi: 10.1016/ j.ijantimicag.2007.07.042

[14] Kokrdová, Z., Pařízek, A., Koucký, M., Pašková, A., Boudová, B. (2016). Septic shock in pregnancy on the basis of an acute pyelonephritis. Ceska Gynekol, 81 (4), 286-288.

[15] Matuszkiewicz-Rowińska, J., Małyszko, J., Wieliczko, M. (2015). State of the art paper Urinary tract infections in pregnancy: old and new unresolved diagnostic and therapeutic problems. Archives of Medical Science, 1, 67-77. doi: 10.5114/aoms.2013.39202

[16] Jepson, R. G., Williams, G., Craig, J. C. (2012). Cranberries for preventing urinary tract infections. Cochrane Database of Systematic Reviews. doi: 10.1002/14651858.cd001321.pub5

[17] Pazos Otero, N., Fuentes Ricoy, L., Ferrández Pérez, B., Martínez Vázquez, C., Martínez Poch, M., Osuna Díaz, J. L. (2007). Pyelonephritis and pregnancy. Our experience in a general hospital. Anales de Medicina Interna, 24 (12), 585-587.

[18] Sheiner, E., Mazor-Drey, E., Levy, A. (2009). Asymptomatic bacteriuria during pregnancy. The Journal of Maternal-Fetal \& Neonatal Medicine, 22 (5), 423-427. doi: 10.1080/14767050802360783

[19] Smaill, F. M., Vazquez, J. C. (2015). Antibiotics for asymptomatic bacteriuria in pregnancy. Cochrane Database of Systematic Reviews. doi: 10.1002/14651858.cd000490.pub3

[20] Ailomazyan, E. K. (1999). Obstetrics. Saint-Petersburg, 850.

\title{
INVESTIGATION OF THE CHEMICAL COMPOSITION AND EFFICIENCY OF SEED OIL OF SPINDLE TREE (EUONYMUS EUROPAEA L.) ON THE MODEL OF NON-ALLERGIC DERMATITIS
}

\author{
Olha Vrubel \\ Department of Pharmacognosy and Botany \\ Danylo Halysky Lviv National Medical University \\ 69 Pekarska str., Lviv, Ukraine, 79010 \\ Igor Nektegaev \\ Department of Pharmacology \\ Danylo Halysky Lviv National Medical University \\ 69 Pekarska str., Lviv, Ukraine, 79010 \\ Volodymyr Antonyuk \\ Department of Pharmceutical chemistry and pharmacognosyFPGE \\ Danylo Halysky Lviv National Medical University \\ 69 Pekarska str., Lviv, Ukraine 79010 \\ Institute of Cell Biology of National Academy of Sciences of Ukraine \\ 14/16 Dragomanova str., Lviv, Ukraine, 79005
}

Abstract

The aim of the research was to study the chemical composition and effectiveness of the seed oil of Spindle tree (Euonymus europaea L.) on the non-allergic contact dermatitis model. 